\title{
Vascular expression of trehalose phosphate synthase1 (TPS1) induces flowering in Arabidopsis
}

\author{
Jorge-Luis Ruiz-Salas ${ }^{1}$, Roberto Ruiz-Medrano ${ }^{1}$, María del Carmen Montes-Horcasitas ${ }^{1}$, Kenny \\ Alejandra Agreda-Laguna ${ }^{1}$, Jesús Hinojosa-Moya ${ }^{2}$, Beatriz Xoconostle-Cázares ${ }^{1}$ *
}

${ }^{1}$ Departamento de Biotecnología y Bioingeniería, Centro de Investigación y de Estudios Avanzados del Instituto Politécnico Nacional. Av. IPN San Pedro Zacatenco, 07360, Ciudad de México

${ }^{2}$ Facultad de Ingeniería Química, Benemérita Universidad Autónoma de Puebla, Campus Acatzingo, Puebla, México

\section{*Correspondence:bxoconos@ cinvestav.mx}

\begin{abstract}
Trehalose and Trehalose-6-phosphate (T6P) have been described as signaling molecules involved in plant growth, development, carbon partitioning, flowering and response to biotic and abiotic stress. In order to investigate the role of T6P in vascular tissue signaling, Arabidopsis, plants expressing the TPS1 gene under the control of the actin depolymerizing factor 3 promoter (At5g59880) were generated by Agroinfiltration. Flower development and silique production occurred earlier in overexpressing lines compared to wild type (WT) control plants, while accumulation of TPS1 transcripts in overexpressing plants was considerably higher in silique, rosette and cauline leaves than in WT plants. Flowering Locus T (FT, AT1g65480) transcript was detected in rosette and cauline leaves in tps 1 overexpressing lines, which could cause the observed early flowering. Trehalose levels were higher in rosette and cauline leaves in TPS1 lines, although no differences were observed in photosynthesis rate. These results suggest that the ectopic expression in the vasculature of the TPS1 gene induced trehalose accumulation in the vasculature, inducing flowering without compromising plant development.
\end{abstract}

Keywords: Trehalose, flowering, growth, vasculature, promoter, Arabidopsis.

Abbreviations: $\mathrm{T}_{6} \mathrm{P}_{-}$Trehalose-6-phosphate; WT_Wild Type; FT_Flowering Locus T; TPS_Trehalose Phosphate Synthase; TPP_ Trehalose phosphate phosphatase; UDPG_UDP-glucose; G6P_glucose-6-phosphate; TRE_Trehalase; CaMV 35S Promoter_ Promoter 35S of the Cauliflower Mosaic Virus; TCTP_ Translationally-Controlled Tumor Protein; CDK_ Cyclin Dependent Kinase; AGPase_ADP-glucose pyrophosphorylase; psbY_photosystem II core complex proteins; RBCS-3B_ Rubisco small subunit; PGK1_ Phosphoglycerate kinase; ALDP1_ Fructose-bisphosphate aldolase.

\section{Introduction}

For many years, trehalose was considered a rare sugar, as it had only been isolated from resurrection plants. More recently, it has been found in most, if not all plants. Indeed, the trehalose pathway has been implicated in the response to biotic and abiotic stress in plants. Trehalose is a non-reducing disaccharide formed by two glucose molecules joined by a glycosidic $\alpha-(1-1)$ bond (Elbein, 1974). In plants, trehalose is synthesized in two steps by trehalose phosphate synthase (TPS) and trehalose phosphate phosphatase (TPP) (Avonce et al., 2005). TPS synthesizes Trehalose-6-phosphate (T6P) from UDP-glucose (UDPG) and glucose-6-phosphate (G6P), which can be hydrolyzed into two glucose monomers by trehalase (TRE). While there is evidence that T6P accumulation confers protection to different types of stress, overexpression of trehalase under the CaMV $35 \mathrm{~S}$ promoter in Arabidopsis caused stomatal closure, increasing the tolerance to water deficit without changes in T6P levels (Van Houtte et al., 2013). Thus, more work is needed to determine the precise role of T6P in whole plant physiology.

Recent work has shown that, besides being an intermediary product in trehalose metabolism, T6P can modulate several processes in plants. Thus, it functions as a mediator of carbon metabolism and, as mentioned before, it accumulates under biotic and abiotic stress. Increased levels of T6P are observed in maize when salt stress is applied; genes controlling trehalose metabolism were more affected under this treatment (Clémence et al., 2015). Interestingly, T6P appears to be involved in carbon signaling in biosynthetic processes regulating plant growth, through the protein kinase SnRK1, a global regulator of metabolism (Nunes et al., 2013). The overexpression of E. coli TPS improves sugar utilization in Arabidopsis, implicating T6P in carbohydrate allocation and growth via the control of the glycolysis pathway (Schluepmann et al., 2003). T6P is also involved in embryo maturation; a tpsl loss-of-function mutation provokes a recessive, embryonic lethal phenotype. It is also indispensable in normal vegetative growth and transition to flowering (Eastmond et al., 2002; Van Dijken et al., 2004). The overexpression of TPS1 in seedlings regulates glucose metabolism and abscisic acid signaling during vegetative development (Gómez et al., 2010; Avonce et al., 2004). Also, exogenously added trehalose inhibits growth of WT seedlings, likely caused by T6P accumulation (Schluepmann et al., 2004). T6P may also have a role in the transition from 
vegetative to flowering stage. Indeed, loss of TPS1 delays flowering in Arabidopsis (Wahl et al., 2013). T6P inhibits the activity of SnRK1, in contrast to other sugars and sugar phosphates in Arabidopsis; this inhibition stimulates the expression of genes involved in growth, in response to sucrose accumulation under sink-limitation conditions (Nunes et al., 2013; Debast et al., 2011). However, overexpression of T6P phosphatase (TPP1) in maize under the OsMads6 promoter (a rice floral promoter) causes a decrease in T6P levels, which in turn increases SnRK1 activity (Nuccio et al., 2015). It has been observed that overexpression of bZIP11 in Arabidopsis, a transcription factor responsible for growth regulation that partially regulates SnRK1 signaling, causes the accumulation of T6P (Delatte et al., 2011). In contrast, inducible overexpression of bZIP11 decreased T6P content in Arabidopsis, resulting in a reprogramming of carbohydrate metabolism; thus, it is possible that bZIP11 is involved in regulating carbon status in plants via modulation of SnRK1 and T6P levels (Ma et al., 2011). Remarkably, Solanum tuberosum tubers expressing $E$. coli TPP caused strong downregulation of SnRK1 as well as two transcripts for proteins that positively regulate growth, namely translationally-controlled tumor protein (TCTP), and a CDK-activating kinase; in contrast T6P levels were decreased (De Bast et al., 2011). Conversely, expression of $E$. coli otsA (TPS) upregulates several genes associated with growth and development (Paul et al., 2009; Debast et al., 2011). T6P regulates the breakdown of starch to sucrose, possibly in response to the demand for sucrose by growing sink organs during the dark period. T6P positively regulates photosynthesis and starch synthesis by activating ADPglucose pyrophosphorylase (AGPase) (Wingler et al., 2000; Kolbe et al., 2005; Lunn et al., 2006; Gómez et al., 2010). There is a correlation between sucrose and T6P levels since exogenous sucrose provokes an increase in the latter; the same effect is observed with the addition of glucose and fructose. Overexpression of the Arabidopsis TPS1 gene in seedlings accelerates germination rates of seeds grown on glucose when compared with wild type plants (WT) (Avonce et al., 2004; Yadav et al., 2014).

T6P is a modulator of photosynthetic capacity through activation of genes such as photosystem II core complex proteins (psbY), Rubisco small subunit (RBCS-3B), Phosphoglycerate kinase (PGK1) and Fructose-bisphosphate aldolase (ALDP1) (Paul and Pellny, 2002; Kondrák et al., 2011). Adenine nucleotide carrier protein (antl) and sucrose synthase (sus3) are upregulated in TPS1 overexpressing plants (Kondrák et al., 2011). Previous studies in which TPS1 has been expressed under the control of constitutive, inducible and tuber-specific promoters (CaMV35S, ABI3, B33, respectively) resulted in abnormal phenotypes in tobacco, rice, potato, tomato and Arabidopsis (Pilon-Smits et al., 1998; Garg et al., 2002; Schluepmann et al., 2003; Gómez et al., 2010). It has been suggested that T6P signaling appears to balance cellular sink activity (low T6P) with cellular growth (high T6P) (Smeekens, 2015).

We tested the hypothesis that the ectopic expression of TPS1 in the Arabidopsis vasculature could affect flowering time by increasing trehalose content in the symplasm. In this study, we describe the expression of the Arabidopsis TPS1 gene driven by the Arabidopsis actin depolymerizing factor 3 gene (At5g59880) promoter, which is expressed in vascular tissue. Parameters such as flowering time, photosynthetic rate and sugar accumulation were evaluated. We observed that plants displayed an accelerated flowering time and emergence of siliques, suggesting that vascular expression
TPS1 trehalose in the vasculature can induce changes in plant developmental processes.

\section{Results}

\section{The Minimal At5g59880 is a vascular specific promoter}

The Arabidopsis promoter At5g59880, including the 5'UTR, drives the expression of the actin depolymerizing factor 3 (ADF3) in vascular tissue (Ruiz-Medrano et al., 2011). In the present work, a minimal promoter version was designed, including $1000 \mathrm{bp}$ of the upstream promoter and $253 \mathrm{bp}$ of the 5'UTR of this gene to direct the expression of a GFPGUS reporter to the vasculature (Fig. 1A). Histochemical analysis of five independent plants revealed the accumulation of GUS reporter in the vasculature, as observed in the leaf veins of all orders from rosette and cauline leaves, as well as in the vasculature of roots, flowers, siliques and stems (Fig. 1B). Cross sections of stem show an intense blue color in the vascular bundles, associated to GUS expression (Fig. 1C). Blue color is absent in parenchyma, indicating that the promoter drives expression specifically in vascular tissue.

\section{Generation of transgenic independent lines overexpressing} TPS1 in the vasculature

In order to target the expression of trehalose phosphate synthase 1 (TPS1) in the vasculature, the At5g59880 minimal promoter was employed to drive the expression of the TPS1 open reading frame, which was retrotranscribed from mRNA. The binary vector employed also contains the bar gene, providing resistance to the herbicide ammonium glufosinate. A number of herbicide resistant plants were obtained and then characterized for further studies. Independent Arabidopsis lines were obtained and autofertilized. Seeds from T1 transformants were then in vitro germinated in the presence of ammonium glufosinate and segregation of the TPS1 transgene was analyzed in the T2 generation. $72 \%$ of seeds germinated in the presence of the herbicide, suggesting that inheritance of the gene is Mendelian. In order to identify Arabidopsis transformants with one insertion in the genome, homozygous plants were analyzed by droplet digital PCR. Two independent lines were selected harboring a single insertion of the TPS1 gene (Suppl. Fig. 1). In the T3 generation, one homozygous plant per independent line was selected on MS plates. TPS1-overexpressing Arabidopsis lines were designated TPS1 L1 and TPS1 L2 and were further characterized in the present work.

\section{Phenotypic changes in homozygous TPS1 lines}

Phenotype of TPS1 L1 and TPS1 L2 lines was analyzed. The development of both vegetative and reproductive tissues revealed interesting characteristics when compared to wild plants. Indeed, the inflorescence stem in TPS1 plants emerged on average 8 days earlier than WT; also, stems were longer. Plants displayed earlier flowering when grown in hydroponic conditions and in pots (Fig. $3 \mathrm{~A}$ and $3 \mathrm{~B}$ ). Bolting occurred at 21 days after germination (dag) in TPS1 lines, while it was observed at 29 dag in WT plants. No significant differences were observed in seed morphology and number of seeds per plant, indicating that precocious flowering in TPS1 expressing plants did not compromise plant reproductive development. Photosynthetic capacity was also measured in TPS1-expressing plants, where no significant differences were observed at constant irradiation; average rates obtained in TPS 1 and WT were $8 \mu \mathrm{mol} \mathrm{CO} \mathrm{CO}^{-2} \mathrm{~s}^{-1}$ and $7.5 \mathrm{CO}_{2} \mu \mathrm{mol}$ 


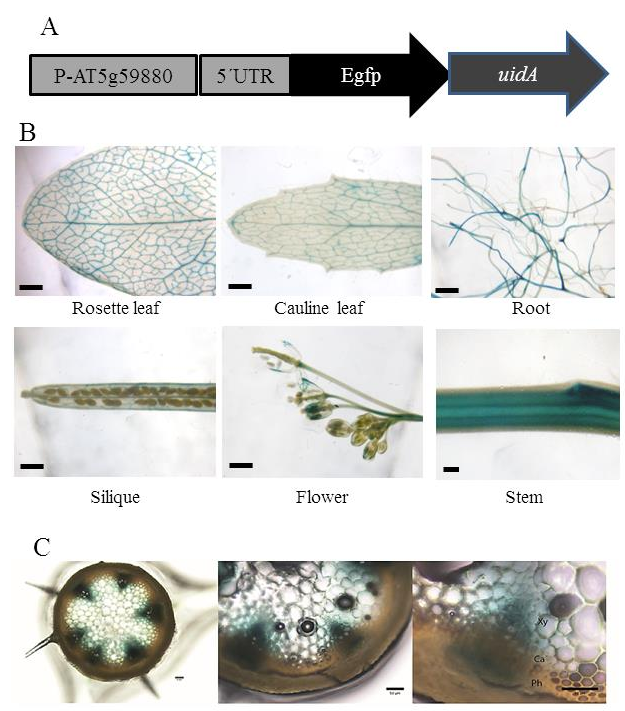

Fig 1. The vascular promoter P-AT5g59880 drives the expression of the reporter gene GUS in veins and vascular bundles. (A) Schematic representation of gene construct employing the vascular promoter P-At5g59880 plus 5' UTR driving the expression of EGFP-uidA. (B) GUS expression in mature rosette leaves, cauline leaves, roots, silique, flower and stem of Arabidopsis plants expressing the construct (bars are $2 \mathrm{~mm}$ ) and (C) Transversal section of stem (bars are $5 \mu \mathrm{m}$ ). Xy, Xylem; Ca, Cambium; Ph, Phloem.

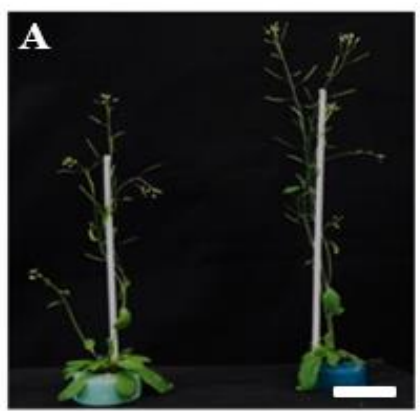

WT

TPS 1

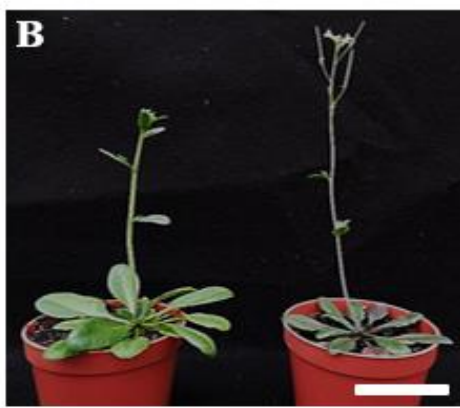

WT
TPS1

Fig 2. Flowering in Arabidopsis TPS1-overexpressing lines occurs earlier than WT plants. 6 week-old plants grown in (A) hydroponic conditions, (B) grown in pots. (Bars are $5 \mathrm{~cm}$ ).
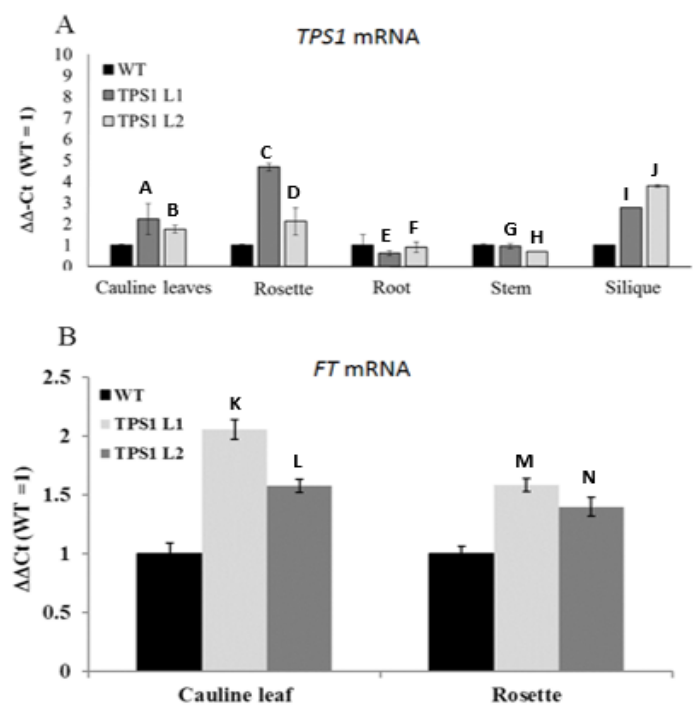

Fig 3. TPS1 mRNA in overexpressing lines accumulates in leaves, stems and siliques. (A) Relative expression of TPS1 gene in TPS1 transgenic plants and wild-type in roots, stems, cauline leaves, rosette leaves and siliques. (B) Relative expression of Flowering Locus T (FT) in TPS1 lines and wild-type in rosette and cauline leaves. Tissues were collected in the afternoon. $\Delta \Delta \mathrm{Ct}$ was calculated employing $18 \mathrm{~S}$ rRNA to normalize the expression. T-student statistical test with similar variance $(\alpha=0.05, \mathrm{n}=3)$ yielded the results: A, 0.158; B, 0.065; C, 0.001; D, 0.127; E, 0.174; F, 0.324; G, 0.382; H, 0.099; I, 0.000; J, 0.000; K, 0.005; L, 0.016; M, 0.006; $\mathrm{N}, 0.035$; where $\mathrm{C}, \mathrm{I}, \mathrm{J}, \mathrm{K}, \mathrm{L}, \mathrm{M}$ and $\mathrm{N}$ were statistically different $(\mathrm{p}<0.05)$. 


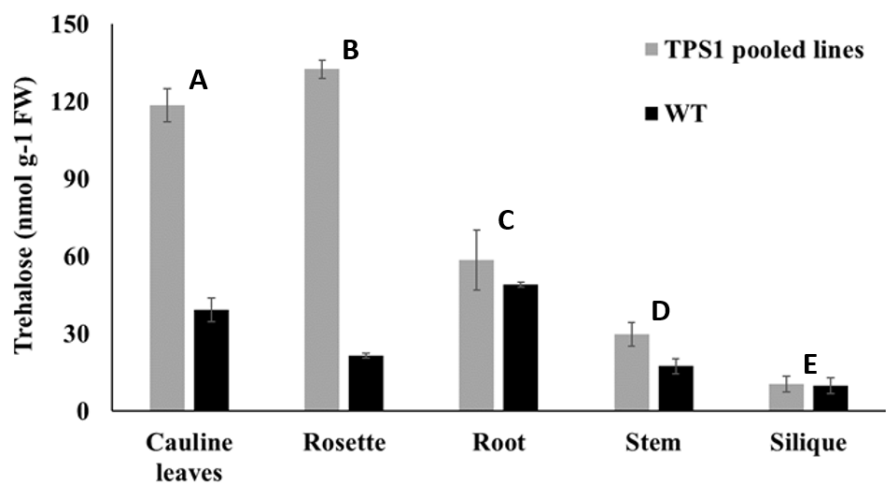

Fig 4. Trehalose accumulates mainly in autotrophic leaves. Trehalose content in TPS1 (pooled L1 and L2) lines compared with WT in roots, stems, cauline leaves, rosette leaves and siliques. T-student statistical test with similar variance $(\alpha=0.05$, $n=3)$ yielded the results: A, 0.004; B, 0.006; C, 0.312; D, 0.048; E, 0.99; where A, B and D were statistically different $(\mathrm{p}<0.05)$.

$\mathrm{m}^{-2} \mathrm{~s}^{-1}$ respectively (Suppl. Fig. 2), no significant statistic differences were obtained when compared with t-student algoritm at $\alpha=0.05$. TPS 1 -expressing lines consistently displayed a darker green color in mature leaves, despite the similar photosynthetic rate at constant radiation.

TPS1 transgenic lines showed higher accumulation of TPS1 mRNA in rosette leaves and siliques, compared with WT

The overexpression of the TPS1 gene was confirmed by quantitative real-time reverse transcriptase PCR (qRT-PCR), employing mRNA from cauline leaves, rosette leaves, roots, stems and siliques from six-week old plants from both WT and TPS1 lines (Fig. 3). The overall endogenous TPS1 mRNA accumulation was similar in all the tested tissues in WT Arabidopsis; however, there were differences in mRNA accumulation in rosette leaves of the TPS1 L1 as well in siliques in both TPS1 lines. T-student statistical test with similar variance $(\alpha=0.05, n=3)$ allowed to assess significant differences. The highest levels of TPS1 mRNA accumulation were in rosette leaves in L1 line, which accumulated threefold higher than WT. Cauline leaves, roots and stem mRNA accumulation was not statistically different when compared with t-student test as shown in Fig. 3A.

\section{Detection of Flowering Locus T mRNA in TPS1 transgenic lines}

As an internal control to assess the reprogramming to early flowering of TPS1 lines, Flowering Locus $T$ (FT) mRNA was selected and quantified, since it has been widely documented to induce flowering in plants through translocation of the protein from mature leaves to the shoot apex via the phloem (Kardailsky et al., 1999; Corbesier et al., 2007; Lin et al., 2007). FT transcripts were detected by qRT-PCR in cauline and rosette leaves in both TPS1-overexpressing lines and WT plants of the same age (six weeks) (Fig. 3B). T-student statistical test with similar variance $(\alpha=0.05, n=3)$ allowed the identification of significant differences in cauline leaves and leaf rosettes. FT mRNA accumulated in cauline leaves of TPS1 L1 and L2, 2 and 1.5 fold levels higher than WT, respectively, while rosette leaves showed an increase of 1.4fold relative to WT plants (Fig. 4B). Basal FT mRNA accumulation was also observed in non-flowering WT, which is consistent with previous reports of the presence of this transcript in all vegetative tissues (Lin et al., 2007).

\section{Trehalose accumulates at higher levels in TPS1 overexpressing Arabidopsis}

The accumulation of trehalose, as a proxy of T6P levels, was quantified in different tissues of transgenic plants employing a sensitive, fluorometric assay (Fig. 4). T-student statistical test with similar variance $(\alpha=0.05, n=3)$ allowed the identification of statistical significance in TPS1 pooled lines in cauline and rosette leaves when compared to WT. While in control plants the highest accumulation was observed in roots, rosette and cauline leaves, TPS1 vascularoverexpressing lines showed an important accumulation of this disaccharide in cauline leaves and rosettes, 2.8 and 6.3 times higher than WT. Indeed, in cauline leaves the content of trehalose in transgenic plants was of $117 \mathrm{nmol} \mathrm{g}^{-1}$ (fresh weight (FW), compared with $42 \mathrm{nmol} \mathrm{g}^{-1}(\mathrm{FW})$ in control plants. In rosette the accumulation of trehalose was $133 \mathrm{nmol}$ $\mathrm{g}^{-1}$ in TPS1 expressing lines, compared to $21 \mathrm{nmol} \mathrm{g}^{-1}$ (FW) in control plants. A slightly higher accumulation was measured in stems while similar trehalose accumulation was observed in roots and siliques (Fig. 4). Thus, overexpression of this gene in vascular tissue leads to increased trehalose content mainly in rosette and cauline leaves.

\section{Discussion}

Some strategies for genetic improvement of crops have focused on manipulating plant productivity by increasing sugar synthesis and mobilization from source to sink tissues (Li et al., 2011; Singh et al., 2011). A complementary approach is to increase levels of certain sugars that act as signaling molecules that may regulate developmental processes in plants such as acceleration of the flowering process and tolerance to both biotic and abiotic stress (Tsai \& Gazzarrini, 2014). The transition from vegetative growth to flowering is highly regulated in plants, depending on both developmental and environmental cues (Corbesier et al., 2007; Lin et al., 2007). Trehalose phosphate synthase 1 (TPS1) and its product, trehalose-6-phosphate (T6P), are considered a key step in the transition from vegetative growth to flowering; it has been described that T6P could activate the transcription of the $F T$ gene in leaves, which is an essential inducer of flowering (Van Dijken et al., 2004; Golembeski et al., 2014; Wahl et al., 2013). Since trehalose is synthesized and accumulates in cytosol, it is reasonable to assume that its accumulation in the vasculature and especially, in the phloem, would lead to its distribution within the whole plant, including those tissues that respond to this molecule. 
Therefore, in the present work we have overexpressed TPS1 from Arabidopsis specifically in the vascular tissue by using a vascular-specific promoter (Ruiz-Medrano et al., 2011), in order to understand its effect in plant development. The vascular tissue has recently emerged as a conduit pivotal for the distribution of signals that integrate plant development and responses to environmental cues (Lucas et al., 2013). The overexpression of TPS1 caused trehalose to accumulate in cauline and rosette leaves, consistent with the autotrophic nature of these tissues. Trehalose could act as a signal molecule promoting flowering, before FT is actually induced by photoperiod in Arabidopsis; this suggests that the trehalose pathway is likely upstream of the known inducers of FT. These findings are in agreement to previous works (Wahl et al., 2013; Dijken et al., 2004). In contrast to previous reports showing that trehalose and T6P play an important role in increasing the photosynthetic capacity of different plant species, we did not detect differences in photosynthetic capacity of TPS1 overexpressing lines and WT plants; however, we measured this capacity at constant irradiation. It will be of interest to test different irradiation levels to identify possible differences in this important physiological parameter. Darker green leaves as those obtained in the TPS1 overexpressing lines, were observed in Nicotiana tabacum expressing TPS1 (otsA) from E. coli and yeast; however, they also accumulated anthocyanin, likely due to physiological stress (Schluepmann et al., 2003). Despite the importance of trehalose and T6P in plant defense and flowering, the expression of the TPS gene employing a strong, constitutive promoter resulted in detrimental phenotypes (Schluepmann et al., 2003; Gómez et al., 2010). Considering that the constitutive expression could mask normal trehalose and T6P performance, the effect of TPS1 expression in the vasculature was studied in this work; indeed, major detrimental effects were not observed in the present case. It is important to consider that Arabidopsis is mostly an apoplastic loader of photoassimilates, as strongly suggested by the disaccharide symporters identified in its genome. However, its capacity of synthesizing sugars in the vasculature (more precisely, in the companion cell) could induce symplasmic translocation of nutrients, thus providing directionality to trehalose from source to sink tissues.

The transition from vegetative growth to flowering is highly regulated in plants, depending on both developmental and environmental cues (Song et al., 2015). T6P can activate the transcription of the FT gene in leaves (Golembeski et al., 2014). We observed that the plants containing the TPS1 construct accelerated the transition from vegetative growth to flowering, compared with the wild type plants; so it is possible that trehalose could be acting as a flowering modulator in this process. This result is in agreement with other works demonstrating that TPS1 is essential for the transition from the vegetative phase to flowering and TPS1; indeed, knock-out of this gene provoked a delay in flowering in Arabidopsis (Van Dijken et al., 2004; Wahl et al., 2013). A high accumulation of trehalose in rosette and cauline leaves was observed, in contrast to siliques, in which the levels of trehalose were similar to WT. Trehalose synthesis can be also induced by abiotic stress as observed in rice; the expression of TPS1 under the control of the rice actin promoter increased trehalose accumulation (Garg et al., 2002; Li et al., 2011). The results presented here indicate that the vascular expression of TPS1 induced early flowering, without compromising plant development, in contrast to TPS1 expression driven by the constitutive CaMV $35 \mathrm{~S}$ promoter in $N$. tabacum, where plants display a stunted phenotype (Schluepmann et al., 2003; Gómez et al., 2010).
Since Arabidopsis display apoplastic loading/unloading of photoassimilates, the expression of TPS1 under the expression of a vascular promoter would not have a major effect on sugar mobilization; however, differential TPS1 mRNA accumulation and trehalose content were observed, thus suggesting that there could be symplasmic trehalose mobilization to reach heterotrophic tissues. The increased accumulation of trehalose and likely T6P in the vasculature, due to ectopic expression of TPS1, could saturate the apoplastic transport route, mediated by symporters and thus limiting the translocation of photoassimilates to heterotrophic tissues. However, a second mobilization route, represented by the symplasm could contribute to bypass the apoplast limitation, now allowing these signaling sugars to reach sink tissues through the vasculature. More investigation is necessary to perform similar assays in plants with symplasmic loading/unloading, to understand the role of trehalose and T6P in orchestrating different roles in the plant development.

\section{Materials and Methods}

Plant material, growth conditions and Arabidopsis genetic transformation

Arabidopsis seeds ecotype Col- 0 were stratified at $4^{\circ} \mathrm{C}$ in the dark for $3 \mathrm{~d}$ and then one seedling per pot was planted and grown at $22^{\circ} \mathrm{C}$ in a greenhouse under controlled conditions. Arabidopsis was genetically transformed by the floral dip method (Zhang et al., 2006) and selected by spraying with $120 \mathrm{mgL}^{-1}$ of the herbicide ammonium glufosinate (Bayer, Germany). $F_{1}$ seeds were harvested and grown on soil and selected with herbicide as described above. Mendelian segregation was analyzed by growing $F_{2}$ seeds on MS medium supplied with $2 \%$ of sucrose and $25 \mu \mathrm{M}$ of ammonium-glufosinate (Sigma-Aldrich, St. Louis MO). Plates were incubated in photoperiod (16-8 h light-dark) under controlled conditions. Herbicide-resistant plants were analyzed by PCR, employing the primers tps1-transgenF and tps1-transgenR (Suppl. Table 1). Two independent lines were obtained; further analyses of homozygous plants were performed in both lines.

\section{Histochemical analysis of GUS driven by the At5g59880 promoter}

Eight independent transgenic plants Arabidopsis plants ecotype Col-0 were used for histochemical studies (RuizMedrano et al., 2011). Full rosette leaves, cauline leaves, stems, roots, flowers and siliques were sectioned and incubated overnight at $37^{\circ} \mathrm{C}$ with GUS buffer and rinsed, as previously described (Weigel \& Glazebrook, 2002). Tissues were examined using a Nikon SMZ745T stereomicroscope (Nikon, Japan), and images collected using a Nikon digital camera model DS Ri1. The GUS reaction was considered negative when no stain was detected after a $24 \mathrm{~h}$ incubation period.

\section{Amplification and assembly of TPS1 construct}

Total RNA was extracted from Arabidopsis WT using an RNeasy Kit (Qiagen Hilden, Germany) following the supplier's instructions. cDNA was synthetized with RTSuperscript III (Invitrogen), using dT-GAGA and SMART primers. The TPS1 ORF (At1g78580) was synthetized from Arabidopsis cDNA using the TPS1-F and TPS1-R primers (Suppl. Table 1). The PCR program used was as follows: 
denaturation at $94^{\circ} \mathrm{C}$ for $3 \mathrm{~min}, 30$ cycles of amplification $\left(94^{\circ} \mathrm{C}, 35 \mathrm{~s} ; 61^{\circ} \mathrm{C}, 35 \mathrm{~s}\right.$ and $\left.72^{\circ} \mathrm{C}, 2 \mathrm{~min} 40 \mathrm{~s}\right)$ followed by 7 min at $72^{\circ} \mathrm{C}$. The minimal vascular promoter from the At5g59880 gene was designed from the amplified promoter described previously (Ruiz-Medrano et al., 2011). The TPS 1 gene flanked by promoter and terminator sequences was in vitro assembled using the assembly-PCR method (GeneSeam Technology), using previously primer-assembled promoter, open reading frame and NOS terminator. The primers used in the construct were 59880-F, 59880TPS1 connect-F, 59880TPS1 connect-R, TNOSTPS1 connect-F and TNOSTPS1 connect-R and TNOS-R (Suppl. Table 1). Flanking primers 59880-F and TNOS-R were employed to PCR-amplify the complete construct and then cloned into the pCR8/GW/TOPO TA vector (Invitrogen, Carlsbad CA). Recombination cloning in destination vector was performed by mixing $100 \mathrm{ng}$ of entry vector with $150 \mathrm{ng}$ of pBGWFS7,0 destination vector (Karimi et al., 2007), $0.5 \mu \mathrm{l}$ of LR-clonase (Invitrogen) and incubated at $24^{\circ} \mathrm{C}$ for $1 \mathrm{~h}$ and then transformed into E. coli. The recombinant destination vector was verified by sequencing with both flanking and internal primers. The binary vector was then transformed into Agrobacterium tumefaciens strain C58C1 by electroporation and transformants selected on LB medium containing Spectinomycin $\left(100 \mathrm{mg} \cdot \mathrm{L}^{-1}\right)$.

\section{Copy number in TPS1 independent lines}

Genomic DNA was extracted from F1 generation of TPS1 transgenic plants using a DNeasy kit (Qiagen). Insertion copy number was estimated by using a droplet digital system (Q100X, BioRad). The target fragment was amplified from the bar gene and the sequence of primers are described in the Suppl. Table 1. The mixture was prepared in a duplex reaction in a total volume of $20 \mu \mathrm{l}, 250 \mathrm{nM}$ of probe and 900 $\mathrm{nM}$ of each primer, HMGB1 (AT3g51880) and BAR, 2x of reaction mixture (ddPCR supermix for probes, BioRad) and 5 ng of genomic DNA. The genomic DNA was previously digested with EcoRI (NEB) for $1 \mathrm{~h}$ at $37^{\circ} \mathrm{C}$ (Mazaika and Homsy, 2014). The program was as follows: $94^{\circ} \mathrm{C}$ during 10 min, after 40 cycles of $94^{\circ} \mathrm{C}$ during $30 \mathrm{~s}$ and $56^{\circ} \mathrm{C}$ for $1 \mathrm{~min}$ and a final extension of $72^{\circ} \mathrm{C}$ for $10 \mathrm{~min}$.

\section{Analysis of photosynthesis and phenotypic profile}

Photosynthesis rate was determined in eight-week old plants grown in special pots adapted to the Arabidopsis device of the Photosynthesis Licor System (LI-6400 XT) provided with a $\mathrm{CO}_{2}$ cartridge at a constant radiation of $400 \mu \mathrm{M} \cdot \mathrm{m}^{-2} \mathrm{~s}^{-1}$. The measurements of flowering time and photosynthesis rate in twenty plants of both WT and TPS1 plants were performed by triplicate and an ANOVA test with Tukey test $(\alpha=0.05)$ was employed to assess possible differences in both WT plants and transformants.

\section{Quantitative RT-PCR}

Tissues from 6 week-old plants were collected from TPS1 overexpressing lines and WT, frozen with liquid nitrogen and total RNA extracted with Direct-zol RNA miniprep kit (Zymo Research, Irvine, CA). Transcript levels were quantified by Real Time RT-qPCR employing the StepOne Plus device (Applied Biosystems, Carlsbad CA). The RTqPCR was normalized using $18 \mathrm{~S}$ with $18 \mathrm{~S}-\mathrm{F}$ and $18 \mathrm{~S}-\mathrm{R}$ primers, while a fragment of the TPS1 gene was amplified with the TPS1-F qPCR and TPS1-R qPCR primers; the $F T$ transcript (At1g65480) was amplified with FT-R qPCR and
FT-F qPCR primers (Suppl. Table 1). Reactions were assayed by triplicate employing three biological samples. The reaction mix was made as follows; KAPPA SYBR FAST Master Mix $(2 \mathrm{x})$, forward primer $(10 \mu \mathrm{M})$, reverse primer $(10$ $\mu \mathrm{M})$, KAPPA RT mix (50x) and 25 ng of RNA. cDNA synthesis was performed at $42^{\circ} \mathrm{C}$ for $5 \mathrm{~min}, 94^{\circ} \mathrm{C}$ to inactivate RT for $5 \mathrm{~min}$, denaturing at $95^{\circ} \mathrm{C}$ for $3 \mathrm{~s}$ and anneal/extend step at $60^{\circ} \mathrm{C}$ for $20 \mathrm{~s}$. Relative mRNA accumulation was determined according to the comparative $\Delta \Delta \mathrm{Ct}$ method (Livak and Schmittgen, 2001).

\section{Analysis of Trehalose accumulation}

Arabidopsis TPS1 (L1 and L2 lines) and WT were grown at $22^{\circ} \mathrm{C}$ and a constant radiation of $400 \mu \mathrm{M} \cdot \mathrm{m}^{-2} \cdot \mathrm{s}^{-1} .50 \mathrm{mg}$ of fresh weight $(\mathrm{FW})$ of 10 week-old was collected from each tissue from a pool of three plants (rosette and cauline leaves, stems, roots and siliques). Plant material was frozen with liquid nitrogen and ground to fine powder with stainless steel beads with a Tissue Lyser equipment (Qiagen) for $90 \mathrm{sec}$. Extract was semipurified with a $30 \mathrm{mg}$ SPE cartridge (Milford, MA, USA) as described by Sastre-Toraño et al. (2012). Trehalose content was estimated using a sensitive fluorometric assay (Carrillo et al., 2013), with minimal modifications. Trehalase was incubated with the extracts at $37^{\circ} \mathrm{C}$ for $15 \mathrm{~min}$ and fluorescence quantified with the Qubit 3.0 Fluorometer (Thermo Fisher Scientific, Waltham MA). Standard curves were generated by triplicate with known trehalose standards (Sigma-Aldrich, St. Louis MO) to infer its concentration in the plant extracts.

\section{Statistical analysis}

T-student statistical test was employed to assess significant differences in TPS1 lines compared to WT. Excel software 2013 was employed for data analyses, utilizing comparison of variables with similar variance with $\alpha=0.05$.

The results presented in this research suggests that the ectopic expression of informational molecules such as the sugar trehalose in the Arabidopsis vascular cells, by expressing TPS1 gene in vascular cells, increased trehalose accumulation in the vasculature, thus inducing flowering without compromising plant development.

\section{Acknowledgements}

This work was supported by a $\mathrm{PhD}$ fellowship provided by CONACyT to JLRS (219416) and KAAL (240193), FINNOVA grant to BXC, and CONACyT grants (105985, 158801 to BXC and 156162 to RRM).

\section{References}

Avonce N, Leyman B, Mascorro-Gallardo OJ, Van Dijck P, Johan M, Thevelein JM, Iturriaga G (2004) The Arabidopsis trehalose-6-P synthase AtTPS1 gene is a regulator of glucose, abscisic acid, and stress signaling. Plant Physiol. 136:36493659.

Avonce N, Leyman B, Thevelein J, Iturriaga G (2005) Trehalose metabolism and glucose sensing in plants. Biochem Soc Trans. 33:276-279.

Carrillo P, Feil R, Gibson Y, Satoh-Nagasawa N, Jackson D, Bläsing O, Stoitt M, Lunn E J (2013) A fluorometric assay for trehalose in the picomole range. Plant Methods. 9:21. 
Clémence H, Bledsoe SW, Griffiths CA, Kollman A, Paul JM, Sakr S, Lagrimini LM (2015) Differential role for trehalose metabolism in salt-stressed maize. Plant Physiol. 169:1072-1089.

Corbesier L, Vincent C, Jang S, Fornara F, Fan Q, Searle I, Giakountis A, Farrona S, Gissot L, Turnbull C, Coupland G (2007) FT protein movement contributes to long-distance signaling in floral induction of Arabidopsis. Science 316:1030-1033.

Debast S, Nunes-Nesi A, Hajirezaei M R, Hofmann J, Sonnewald U, Fernie AR, Börnke F (2011) Altering trehalose-6-phosphate content in transgenic potato tubers affects tuber growth and alters responsiveness to hormones during sprouting. Plant Physiol. 156:1754-1771.

Delatte TL, Sedijani P, Kondou Y, Matsui M, de Jong G, Somsen GW, Wiese-Klinkenberg A, Primavesi LF, Paul MJ, Schluepmann H (2011) Growth arrest by trehalose-6phosphate: an astonishing case of primary metabolite control over growth by way of the snrk1 signaling pathway. Plant Physiol. 157:160-74.

Eastmond PJ, Van Dijken AJH, Spielman M, Kerr A, Tissier AF, Dickinson HG, Jones, JDG, Smeekens SC, Graham IA (2002) Trehalose-6-phosphate synthase 1, which catalyses the first step in trehalose synthesis, is essential for Arabidopsis embryo maturation. Plant J. 29:225-235.

Elbein AD (1974) The metabolism of trehalose. Adv Carbohydr Chem \& Biochem. 30: 227-256.

Gómez LD, Gilday A, Feil R, Lunn JE, Graham IA (2010) AtTPS1-mediated trehalose 6-phosphate synthesis is essential for embryogenic and vegetative growth and responsiveness to $\mathrm{ABA}$ in germinating seeds and stomatal guard cells. The Plant J. 64: 1-13.

Garg AK, Kim JK, Owens TG, Ranwalas AP, Choi YD, Kochian LV, Wu RJ (2002). Trehalose accumulation in rice plants confers high tolerance levels to different abiotic stresses. Proc Natl Acad Sci 99:15898-15903.

Golembeski GS, Kinmonth-Schults HA, Song YH, Imaizumi $\mathrm{T}$ (2014) Photoperiodic flowering regulation in Arabidopsis thaliana. Adv Bot Res. 72:1-28.

Kardailsky I, Shukla VK, Ahn JH, Dagenais N, Christensen SK, Nguyen JT, Chory J, Harrison MJ, Weigel D (1999) Activation tagging of the floral inducer FT. Science. 286:1962-1965.

Karimi M, Depicker A, Pierre H (2007) Recombinational cloning with plant gateway vectors. Plant Physiol. 145:1144-1154.

Kolbe A, Tiesse, A, Schluepmann H, Paul M, Ulrich S, Geigenberger P (2005) Trehalose 6-phosphate regulates starch synthesis via posttranslational redox activation of ADP-glucose pyrophosphorylase. Proc Nat Acad Sci 201:11118-11123.

Kondrák M, Marincs F, Kalapos B, Juhász Z, Bánfalvi Z (2011) Transcriptome analysis of potato leaves expressing the Trehalose-6-Phosphate Synthase 1 Gene of Yeast. PLos One. 6:e23466.

Li HW, Zang BS, Deng XW, Wang XP (2011) Overexpression of the trehalose 6-phosphate synthase gene OsTPS1 enhances abiotic stress tolerance in rice. Planta. 234:1007-1018.

Lin M-K, Belanger Lee Y-J, Varkonyi-Gasic E, Taoka K-I, Miura E, Xoconostle-Cázares B, Gendler K, Jorgensen RA, Phinney B, Lough TJ, Lucas WJ (2007) FLOWERING LOCUS $\mathrm{T}$ protein may act as the long-distance florigenic signal in the cucurbits plant Cell. 19:1488-1506.

Livak KJ, Schmittgen TD (2001) Analysis of relative gene expression data using real-time quantitative PCR and the 2(-Delta Delta C (T)) Method. Methods. 25:402-408.
Lunn JE, Feil R, Hendriks JHM, Gibon Y, Morcuende R, Osuna

Scheible WR, Carrillo P, Hajirezaei MR, Stitt M (2006) Sugar-induced increases in trehalose 6-phosphate are correlated with redox activation of ADP glucose pyrophosphorylase and higher rates of starch synthesis in Arabidopsis thaliana. Biochem J. 397:139-148.

Ma J, Hanssen M, Lundgren K, Hernández L, Delatte T, Ehlert A, Liu CM, Schluepmann H, Dröge-Laser W, Moritz T, Smeekens S, Hanson J (2011) The sucrose regulated Arabidopsis transcription factor bZIP11 reprograms metabolism and regulates trehalose metabolism. New Phytol. 191:733-745.

Mazaika E, Homsy J (2014) Digital Droplet PCR: CNV Analysis and Other Applications. Curr Protoc Hum Genet. 82:7.24.1-7.24-13.

Nuccio ML, Wu J, Mowers R, Zhou HP, Meghji M, Primavesi LF, Paul MJ, Chen X, Gao Y, Haque E, Basu SS, Lagrimini LM (2015) Expression of trehalose-6phosphate phosphatase in maize ears improves yield in well-watered and drought conditions. Nat Biotechnol. 33:862-869.

Nunes C, O`Hara LE, Primavesi LF, Schluepmann H, Somse GW, Silva AB, Favereiro PS, Wingler A, Paul MJ (2013) The trehalose 6-phosphate/SnRK1 signaling pathway primes growth recovery following relief of sink limitation. Plant Physiol. 3:1720-1732.

Paul MJ, Pellny TK (2002) Carbon metabolite feedback regulation of leaf photosynthesis and development. J Exp Bot. 54:539-547.

Paul MJ, Jhurreea D, Zhang Y, Primavesi LF, Delatte T, Schluepmann H, Astrid WA, (2009) Upregulation of biosynthetic processes associated with growth by trehalose 6-phosphate. Plant Signal Behav. 5:386-392.

Pilon-Smits EAH, Terry N, Sears T, Kim H, Zayed A, Hwang S, van Dun K, Voogd E, Verwoerd TC, Krutwagen RW, Goddijn OJM (1998) Trehalose-producing transgenic tobacco plants show improved growth performance under drought stress. J Plant Physiol. 152:525-532.

Ruiz-Medrano R, Xoconostle-Cázares B, Ham BK, Li G, Lucas WJ (2011) Vascular expression in Arabidopsis is predicted by the frequency of CT/GA-rich repeats in gene promoters. Plant J. 67:130-144.

Sastre-Toraño SJ, Delatte TL, Schluepmann H, Smeekens SC, de Jong GJ, Somsen GW (2012) Determination of trehalose-6-phosphate in Arabidopsis thaliana seedlings by hydrophilic-interaction liquid chromatography-mass spectrometry. Anal Bioanal Chem. 402:1353-1360.

Schluepmann H, Pellny T, van Dijken A, Smeekens S, Paul M (2003) Trehalose 6-phosphate is indispensable for carbohydrate utilization and Growth in Arabidopsis thaliana. Proc Natl Acad Sci. 100:6849-6854.

Schluepmann H, van Dijken A, Aghdasi M, Wobbes B, Paul M, Smeekens S (2004) Trehalose mediated growth inhibition of Arabidopsis seedlings is due to trehalose-6phosphate accumulation. Plant Physiol. 13:879-890.

Singh V, Louis J, Ayre BG, Reese JC, Shah J (2011) TREHALOSE PHOSPHATE SYNTHASE 11-dependent trehalose metabolism promotes Arabidopsis thaliana defense against the phloem-feeding insect Myzus persicae. Plant J. 67:94-104.

Smeekens S (2015) From leaf to kernel: trehalose-6phosphate signaling moves carbon in the field. Plant Phys. 169:2-913. 
Song YH, Shim JS, Kinmonth-Schultz HA, Imaizumi T (2015) Photoperiodic flowering: time measurement mechanisms in leaves. Annu Rev Plant Biol. 66:441-464.

Tsai AYT, Gazarrinni S (2014) Trehalose-6-phosphate and SnRK1 kinases in plant development and signaling: the emerging picture. Front Plant Sci. 5:119.

Van Dijken AJH, Schluepmann H, Smeekens SCM (2004) Arabidopsis trehalose-6-phosphate synthase 1 is essential for normal vegetative growth and transition to flowering. Plant Physiol. 135:969-977.

Van Houtte H, Vandesteene L, López-Galvis L, Lemmens L, Kissel E, Carpentier S, Feil R, Avonce N, Beeckman T, Lunn JE, Van Dijck P (2013) Overexpression of the trehalase gene attre 1 leads to increased drought stress tolerance in Arabidopsis and is involved in abscisic acidinduced stomatal closure. Plant Phys. 3:1158-1171.

Wahl V, Ponnu J, Schlereth A, Arrivault S, Langenecker T, Franke A, Feil R, Lunn JE, Stitt M, Schmid M (2013) Regulation of Flowering by Trehalose-6-Phosphate signaling in Arabidopsis thaliana. Science 339:704.
Weigel D, Glazebrook J (2002) Arabidopsis: A Laboratory Manual. Cold Spring Harbor, NY: Cold Spring Harbor Laboratory Press.

Wingler A, Fritzius T, Wiemken A, Boller T, Aeschbacher RA (2000) Trehalose induces the ADP-glucose pyrophosphorylase gene ApL3 and starch synthesis in Arabidopsis. Plant Physiol. 124:105-114.

Yadav UP, Ivakov A, Feil R, Guang You Duan YG, Walther D, Giavalisco P, Piques M, Carillo P, Hubberten HMH, Stitt M, Lunn JE (2014) The sucrose-trehalose 6 phosphate (Tre-6P) nexus: specificity and mechanism of sucrose signaling by Tre6P. J Exp Bot. 65:1051-1068.

Zhang X, Henriques R, Li SS, Qi-Wen N, Chua NH (2006) Agrobacterium-mediated transformation of Arabidopsis thaliana using the floral dip method. Nat Protoc. 1:641-646. 